Gazi University
Journal of Science
http://dergipark.gov.tr/gujs

\title{
Occurrence of a Swirling Vortex without an Intake
}

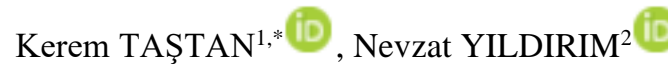 \\ ${ }^{I}$ Gazi University Faculty of Engineering, Department of Civil Engineering, Maltepe, 06570, Ankara, Turkey \\ ${ }^{2}$ Çankaya University Faculty of Engineering, Department of Civil Engineering, Etimesgut, 06790, Ankara, Turkey \\ Highlights \\ - This paper focuses on the mechanics causing the occurrence of a swirling vortex without an intake \\ - Main mechanism of development of the swirling vortex filament is identical for all cases. \\ - Occurrence of a tornado is explained.
}

\section{Article Info}

Received: $16 / 03 / 2020$

Accepted: 01/09/2020

Keywords

Air-core vortex

Dust devil

Swirling vortex flow

Tornado

\begin{abstract}
In this study, mechanisms that cause the occurrence of a swirling vortex without an intake are explained. There are more than one different reasons which may cause the occurrence of an aircore vortex or a swirling-vortex flow filament within an ambient fluid. An air-core vortex or a swirling vortex flow filament can also occur in an ambient fluid media without an intake provided that the coaxial subsurface depressions and thereby the imaginary subsurface spherical sink surface sectors are hydraulically developed by any means (such as rotation of the fluid mass of spherical sector-form, impinging or penetrating jet flow, continuously entering solid body or string etc.). Although studies related to occurrence of a swirling vortex in case of an intake can be found in available literature, there are currently no studies which explains the occurrence mechanisms of a swirling vortex without an intake in detail. In this study, it is showed that the causes for the occurrence of the air-core vortex or the swirling vortex filament can be different but the main mechanism of development of the air-core vortex or the swirling vortex filament is identical for all cases.
\end{abstract}

\section{INTRODUCTION}

Hydraulic engineers frequently discuss the effects and importance of the occurrence of an air-entraining vortex, subsurface vorticies and swirling-vortex flow filament (Figure 1). Occurrence of a swirling vortex in fluid media with an intake is explained in the study by Taştan and Yıldırım [1], Yıldırım et al. [2], and Taştan and Yıldırım [3] due to which it is not repeated herein. In practice, air-core vortex or swirling vortex flow filament occuring in a fluid media without an intake is also frequently encountered (for example, a tornado-swirling or a dust devil-swirling or a fire smoke-swirling, etc.). Although there are several studies relating to the air-entraining vortex occurring at an intake, to authors' knowledge there is not a detailed study relating to the occurrences of air-core vortices, and swirling vortex flow filament in a fluid media without an intake.

Studies relating to the swirling vortices occurring in a fluid media are Deny [4], Anwar et al. [5], Jain et al. [6], Gulliver and Rindels [7], Yıldırım and Kocabaş [8], Kocabaş and Yıldırım [9], Yıldırım et al. [10], Taştan and Yıldırım [11], Sarkardeh et al. [12], Naderi et al. [13], Suerich-Gulick et al. [14], and Taştan [15]. These studies mainly considered the critical submergence $\left[S=S_{c}\right.$ submergence at which the lower end (tip) of the air-core vortex just reaches the intake, herein, $S=$ submergence, $S_{c}=$ critical submergence of an intake] rather than the occurrence of the swirling vortex flow-filament itself and they did not give explanations about how the swirling vortex flow filament develops. 


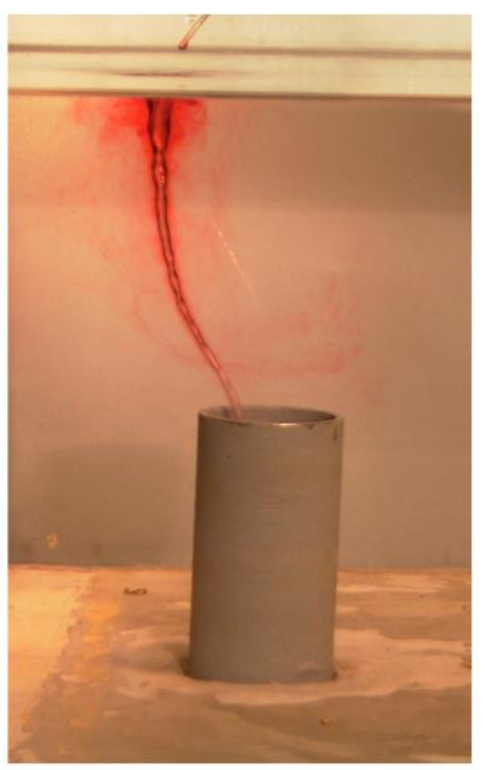

(a)

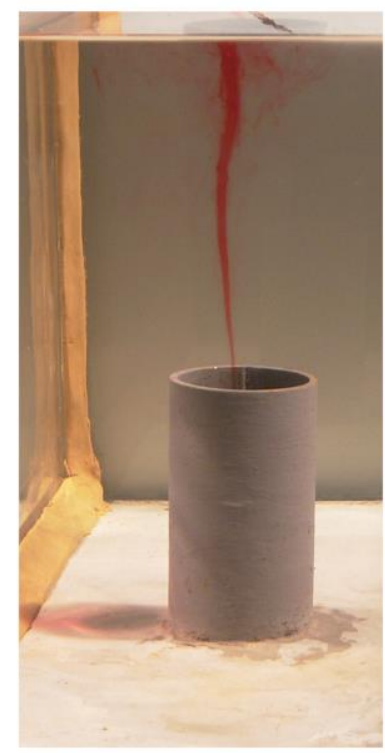

(b)

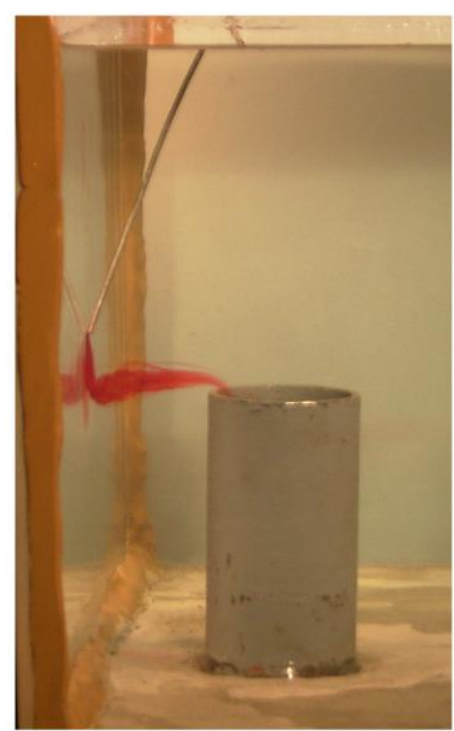

(c)

Figure 1. Different types of vortices occurring at a vertical intake: a) Air-entraining vortex, b) Swirling vortex (no air-core), c) Sub-surface vortex

In the present study, the development mechanism of the swirling vortex flow filament occurring in a fluid media without an intake is examined. The causes for the occurrence of the air-core vortex or the swirling vortex filament can be different but the main mechanism of development of the air-core vortex or the swirling vortex filament is identical for all cases. There exists one common physical mechanism for the development of the air-core vortex and the swirling vortex flow filament occurring in an ambient fluid media with or without an intake. That is as follows.

Yıldırım and Kocabaş [8] and Kocabaş and Yıldırım [9] showed that in case of an intake flow, there exists spherical sink surfaces (SSS), having the same center and the discharge as the intake, within the flow field (Figure 2a). At critical condition, which is defined as the air just enters the intake through an air-entraining vortex, spherical sink surface is named as critical sink surface (CSSS) with a radius of $S_{c}$ (Figure $2 \mathrm{~b}$ ).

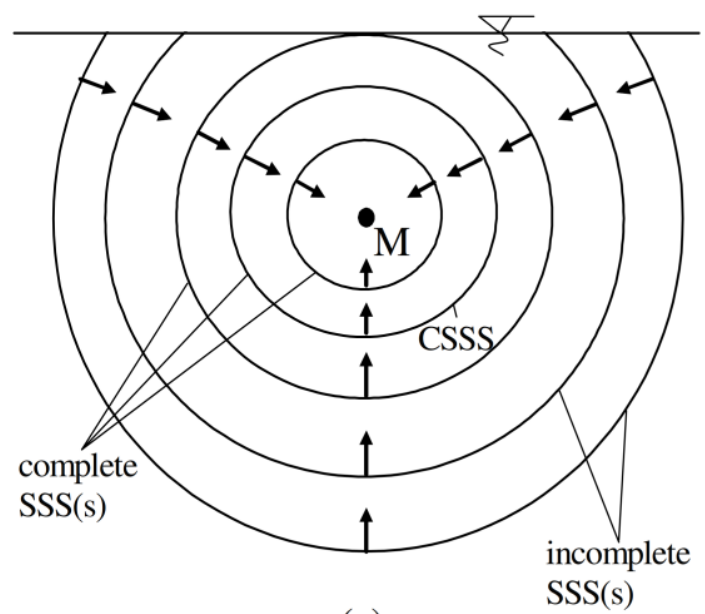

(a)

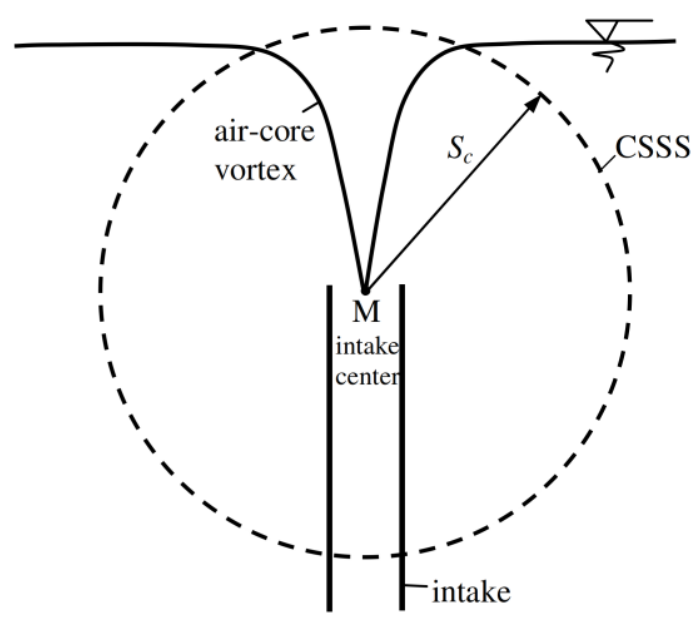

(b)

Figure 2. a) Spherical sink surfaces (SSSs) for an intake, b) Critical spherical sink surface (CSSS) 
A swirling vortex flow filament occurs within an ambient fluid media without an intake as the coaxial subsurface depressions (SSD) (Figure 3) of spherical sink flow characteristics and thereby the imaginary subsurface spherical sink surface sectors (SSSs) (become exist as the ambient fluid fills in the SSDs) are hydraulically developed by any means (such as, rotation of the fluid mass of spherical sector-form, impinging or penetrating jet flow, continuously penetration of fast solid body or string etc.).

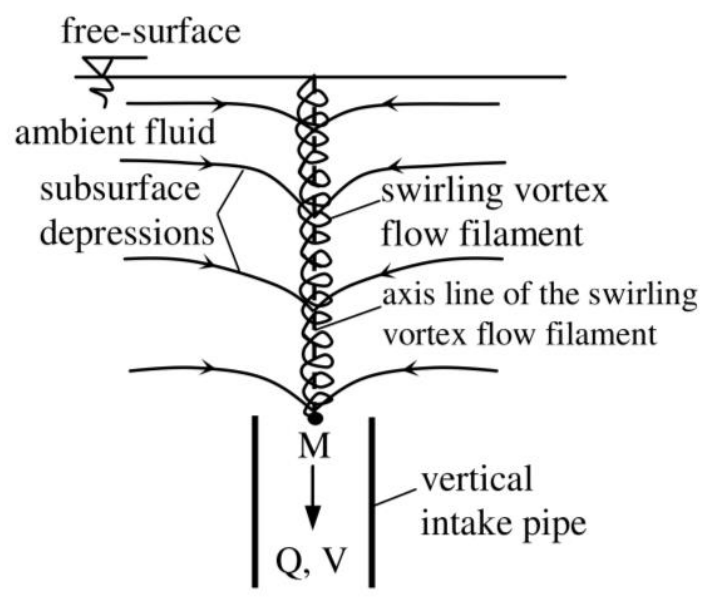

Figure 3. SSDs and the swirling vortex filament [3]

\section{OCCURRENCE OF AN AIR-CORE VORTEX OR SWIRLING VORTEX FILAMENT WITHOUT AN INTAKE}

Taştan and Y1ldırm [3] gives the following explanation for the occurrence of a swirling vortex filament. "So far, in the available literature mentioned earlier, the following two classical points are considered for the potential flow region: i) The circulatory velocity vectors are tangential to completely closed co-centric circular streamlines in a horizontal plane and varying in magnitude inversely with the radial distance from the vertical axis of the vortex (the center of the circular streamlines). The pressure distribution is hydrostatic. The entire ambient circulatory flow with or without an intake is in the form of an infinite number of concurrently rotating co-axial vertical cylindrical sink surfaces extending from the free surface to the bottom boundary and having the same vertical axis as the air-entraining vortex. ii) The path of each particle is a completely closed circular streamline with no swirling, and the entire profile of the air-core vortex is of a single closed-ended "hyperbolic paraboloid of revolution of circular cross-section" with constant flux of magnitude of vorticity (the net circulation is identical at all levels along the entire length of the air-core vortex). However, in reality, these are not the cases. The streamlines are not completely closed circular lines in the horizontal plane and they also have curvatures in the vertical plane. Therefore, the pressure distribution is not exactly hydrostatic. As explained a little later, the circulatory ambient fluid is not in the form of rotating co-centric cylindrical sink surfaces and the circulation along the vortex is not an exactly identical constant. The circulation of the vortex increases from its minimum magnitude at the free surface to its maximum magnitude at the end of the swirling vortex filament."

In the studies by Taştan and Yıldırım [3,11] relating to the air-core vortex at an intake, it is proven that regardless of the flow and geometrical conditions, there exists infinite number of coaxial SSDs of pointsink character that hydraulically developed within the ambient fluid. Each SSD behaves as a depression intake of spherical sink (point-sink) characteristics. It is essential that a fluid mass of spherical sector rotating about its vertical axis under the effects of circulation (that may be self-developed due to the Earth's rotation or circulation) must exist.

Taştan and Yildırım [11] indicated that, as each fluid element on the streamline that is tangent to the maximum velocities moves towards to intake, they drag other particles due to viscosity. This causes an 
elongation (or depression) of SSD(s). These SSD(s) can be considered as depression intake for the ambient fluid filling them out as in the form of a fast jet.

It is understood that development of the swirling vortex is basically the presence of an impinging fast jet flow and thereby the development of coaxial SSD(s) rotating under the effects of Earth's rotation and circulation. For the development of the swirling vortex filament, the presence of an intake may not be the only condition. This mechanism may also be created by several means without an intake.

Without an intake flow, an air-core vortex or swirling vortex can develop within an ambient fluid if at least one of the following five conditions is met (Figure 4).

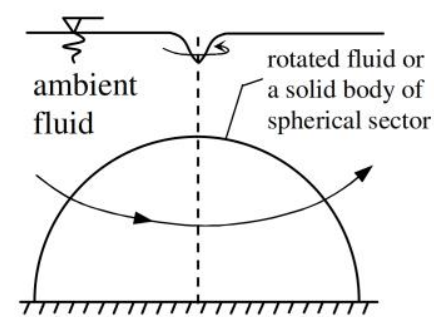

(a)

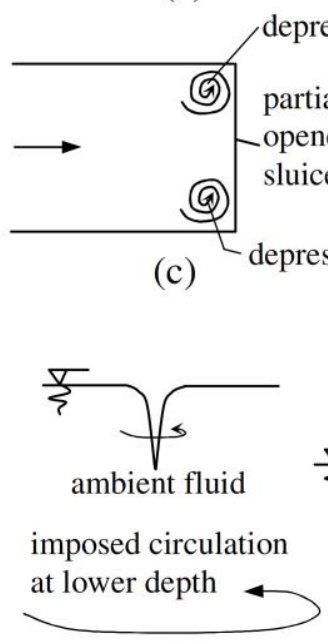

(e)

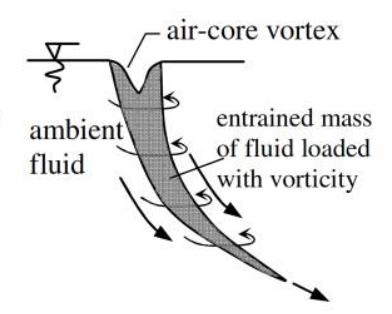

(b)

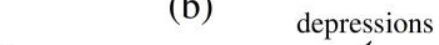

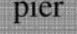

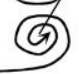

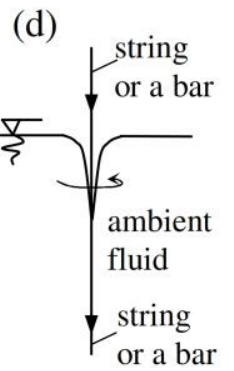

(g)

Figure 4. Development of air-core vortex in a fluid medium without an intake

i) There exists a mass of fluid (or solid object) of a spherical sector rotated about its vertical axis due to the imposed or induced circulation (for example, the occurrence of a tornado, Figures 4a-b); ii) An imposed or induced circulatory fluid section is entrained or dragged or carried through an ambient fluid [for example, locally imposed or induced highly concentrated vorticity-carrying fluid section(s) with and without a converging current may be observed either as a swirling-vortex flow filament or an air-core vortex (i.e., separation vortices, eddies formed due to sharp corners, and piers, and so on; Figures $4 \mathrm{c}-\mathrm{d}]$; iii) An imposed or induced circulation of sufficient magnitude may be applied at the lower section of the depth due to which the centrifugal force may overcome the pressure and capillary forces, resulting in the development of the widening SSD(s) at the lower section of the depth of the fluid, which may need to be required to be filled out by the fluid in the upper section of the depth; therefore if it is insufficient to fill out these widening SSD(s) out, the development of an air-core vortex (dimple) can be observed at the free surface; see Figure 4e (also Levi, [16], Figure 7); iv) A jet of fluid penetrating the free surface and advancing through the ambient fluid; see Figure 4f (also Levi, [16], Figures 8 and 9); and v) A solid string or bar vertically penetrating the free surface and continuously moving vertically downward through the ambient fluid (Figure 4g).

For example for the occurrence of a fire smoke-swirl; an upward rising impinging jet of hot-gas (smoke) issuing from the fire source as a result of high buoyancy force a circulation (due to Coriolis force and other 
disturbances) should be applied to the mass of air or smoke of spherical sector (spherical sectors of airmass should be rotated or spinned about its vertical axis) in a air or smoke media without an intake.

Similarly, as indicated in Figure 5; a swirling dust-devil is the result of rotation of mass of gas of spherical sectors hydraulically developed as the upward impinging hot-gas jet drags the ambient gas along and thereby causes SSDs and SSSs (upward hot-gas jet flow causes SSDs or depression intake for the ambient fluid thereby the collapsing coaxial spherical sectors develop and rotate due to the effects of a net circulation attributed to the sum of the forces due to Earth's rotation, circulations, and the disturbances present within the ambient fluid due to which the twisting or swirling vortex filament flow occurs).

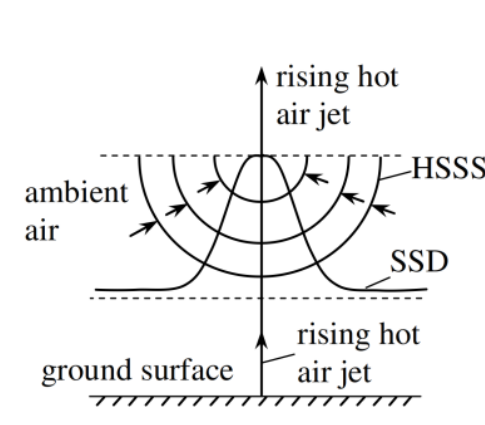

(a)

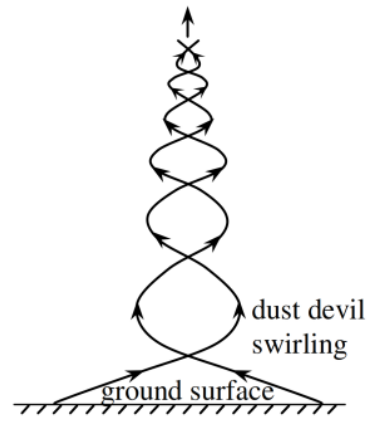

(b)

Figure 5. Development of a dust-devil

In the case of no intake flow, study by Yildırım et al. [11] indicated that under the effects of a net circulation attributed to the sum of the forces due to Earth's rotation, and the disturbances present within the ambient fluid, an air-core vortex or a swirling-vortex flow filament can also develop within an ambient fluid. The surface of the fluid mass through which the net discharge is determined by the net circulation is that of an imaginary sphere (it is an imaginary spherical surface) and $S_{c}$ can be computed from the formula given below (by merging the known and unknown constants into $V_{c}$ )

$A_{c} V_{c}=B \Gamma ;$ or $\frac{A_{c}}{B^{2}} V_{c}=\frac{\Gamma}{B}$

in which $A_{c}$ is the net effective surface area of the critical spherical sink surface (CSSS) (that is the SSSS with radius of $S=S_{c}$ ), $B$ is the characteristic length (i.e. height) of the circulatory flow, $\Gamma$ is the net circulation, and $V_{c}$ is the normal velocity at the CSSS. For a complete CSSS, $A_{c}=4 \pi S_{c}^{2}$. Equation (1) shows that the imposed or induced circulation, including the Coriolis effect, acts and causes the development of the depression intakes (DIs), and the vacuuming action, which is the same as that due to the intake discharge for a mass of fluid or gas that has the form of a complete sphere or a spherical sector. This means that without an intake discharge, an air-core vortex or a swirling vortex can also develop in a mass of fluid or gas in the form of a sphere or a spherical sector that is rotated about its vertical axis.

It worths to consider the occurrences of a tornado. That is as follows.

\section{OCCURRENCE OF A TORNADO-SWIRL}

A "tornado" that has touched down on the ground is, in reality, a swirling vortex flow filament (without an intake flow) occurring within a complete or incomplete spherical sector of a mass of air that is rotated about its vertical axis by another air stream(s). The swirling flow related to a tornado develops in the same manner as explained earlier for the presence of an intake.

Consider a fluid mass of hemispherical form that is rotated by an air stream (wind) about its vertical axis, as indicated in Figures $6 \mathrm{a}$ and $\mathrm{b}$. 


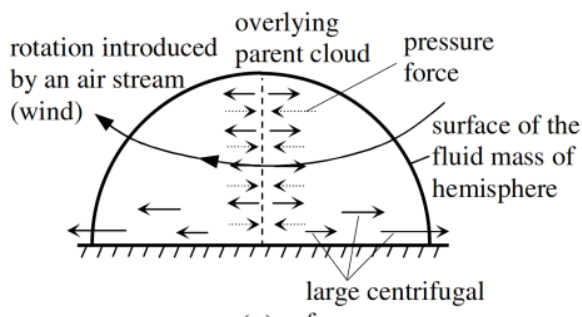

(a) force

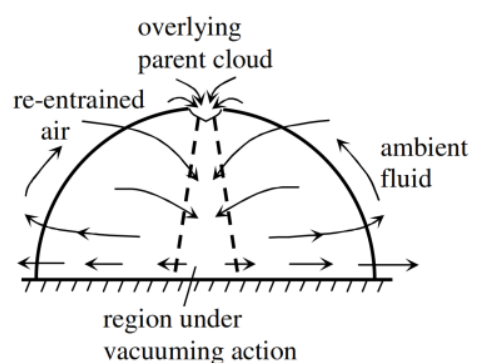

(b)

overlying parent cloud particles filling out the depression

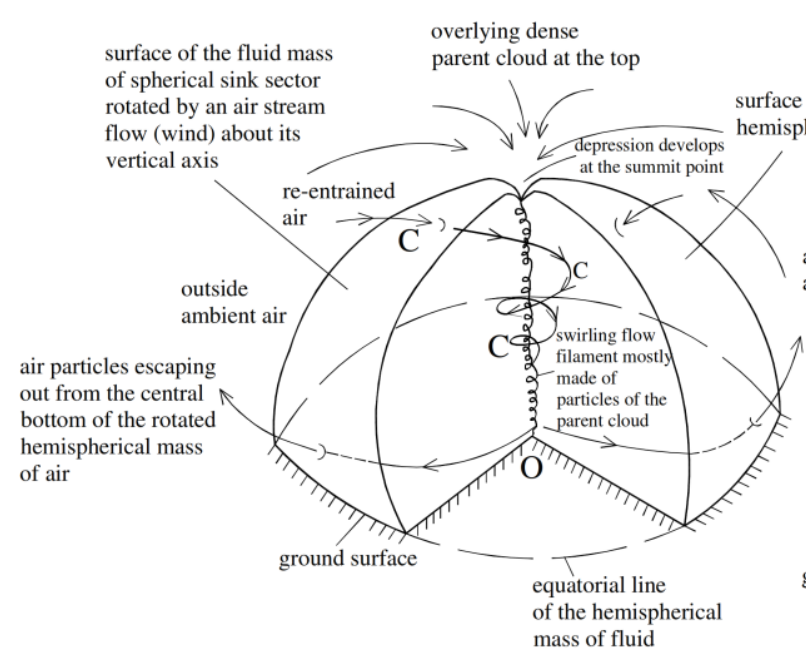

(c)

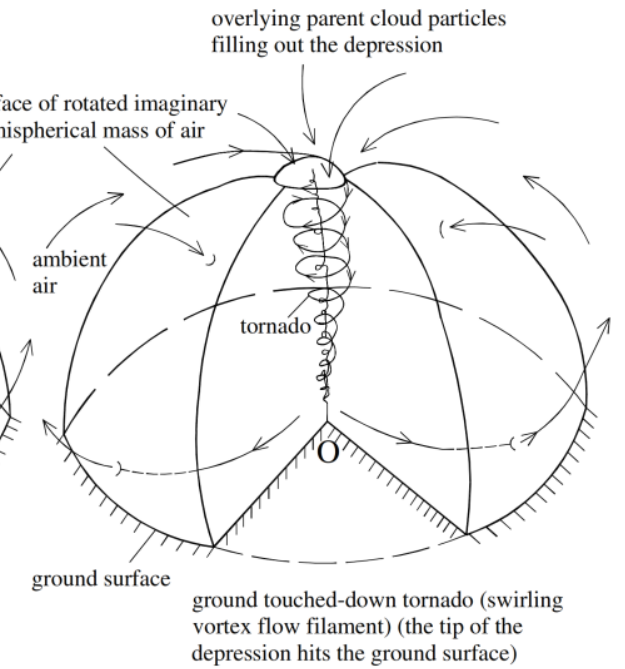

(d)

Figure 6. Development stages of a ground-touched-down tornado

All air particles are under the effects of pressure force and the centrifugal force (attributed to the circulation), which act in the opposite directions. As explained earlier, the circulation increases from the summit point to the base circumference or equatorial line of the hemispherical air mass. If the centrifugal force becomes larger than the pressure force, especially the particles at and close to the bottom, they try to move away from the central region and leave an empty space (suction zone) behind themselves, which is filled out by the point-sink character air flow at higher elevations [very similar to the flow filling in the $\operatorname{SSD}(s)$ of point-sink characters, as in the presence of an intake flow], where the centrifugal force is less than the pressure force. As a result, a type of air flow and a depression develop at the summit point of the hemispherical fluid mass, as indicated in Figure 6b. The depression at the summit point of the hemispherical fluid mass is then filled out by both the ambient fluid and the overlying parent cloud above it. The particles escaping through the surface of the hemispherical fluid mass at and about the lower sections of the hemisphere are re-entrained to the hemisphere through its upper section and fill in the depression (Figures $6 b-c)$. If the circulation at every level of the hemisphere is sufficiently large so that the centrifugal forces are larger than the pressure and capillary forces along the central vertical axis all the way from the summit point to the bottom base of the hemispherical fluid mass, coaxial SSD(s) of point-sink character develop (filled in with the ambient fluid, and the overlying dense parent-cloud particles), extending from the summit point to the bottom base of the hemispherical fluid mass which appears as a swirling vortex (tornado that has touched down on the ground), as presented in Figure 6d. Note that the same types of SSS(s) and their characters as explained for the $\operatorname{SSD}(\mathrm{s})$ in the case of an intake are also present for the flow through the depression(s) in the case of a tornado. The tornado becomes more visible as the dense cloud overlying the lower section of the air mass fills in the depression developed within this rotating hemispherical air mass.

For a tornado that has touched down on the ground, $B=S_{c}=$ the elevation of the bottom of the overlying dense parent cloud with respect to the ground surface. If the land surface is approximately horizontal (not 
a necessary condition), $A_{c}=2 \pi S_{c}^{2}$ (with CSSS being a hemisphere). For a tornado that has touched down on the ground, Equation (1) can be rewritten (by merging the constants into redefined $V_{c}$ ) as;

$V_{c}=\frac{\Gamma}{B}$.

By using regional historical recorded data relating to $B$ and $\Gamma$, it may be possible to approximate the magnitude of the $V_{c}$ from Equation (2) for that specific region. Then, by means of Equation (2), one may approximate the possible occurrences of the local tornados that have touched down on the ground for future storms by measuring or evaluating the real-time magnitudes of $\Gamma$ or $B$ during the storm(s). Equation (1) or (2) indicates that if there is no imposed or induced circulation (including the Coriolis effect, and $\Gamma=0$ ), no air-core vortex or swirling vortex occurs.

Equations (1) or (2) suggests that, in cases of with or without an intake, the occurrence of a tornado or a swirling-vortex flow filament can be eliminated if the development of the suction effect or SSD(s) within the rotating mass of fluid (or gas) in the form of an SSSS is avoided (make $V_{c}$ zero or outwards from the surface of SSSS). This may be done by means of a few methods (some of which may not be practical), as follows.

i) Impose or apply an opposite circulation equal to the existing circulation or create highly turbulent flow within the vortex region to spoil the point-sink characters of the $\operatorname{SSD}(\mathrm{s})$ and reduce the net circulation (this may not be practical for a tornado).

ii) Pump, import, or inject a sufficient amount of fluid or gas brought from a far region (a distance a few times larger than $B=S_{c}$ ) into the vortex core region.

iii) Suddenly release a sufficiently large amount of air (compressed and stored gas in advance) into the vortex region.

iv) Continuously release a sufficiently amount of gas from one or more than one source within the vortex region where the tornado is likely to occur.

v) At a small vertical distance from the ground surface and several places far from the center of the tornado along the circumference (preferably along the equatorial circumference) of the tornado, introduce several gas jets tangential to the rotating mass of the fluid in the opposite direction of rotation to the tornado (vortex) to create an opposite circulation to reduce or even eliminate the net circulation of the tornado or vortex [and thus eliminate the development of $\operatorname{SSD}(\mathrm{s})]$.

vi) Others (i.e., horizontal radial vanes).

\section{CONCLUSIONS}

The following conclusions were obtained from the present study.

- The causes for the occurrence of the swirling vortex filament can be different but the main mechanism of development of the air-core vortex or the swirling vortex filament in a fluid media with and without intake is identical for all cases.

- The swirling vortex filament in a fluid media without an intake is the result of collapses and rotations of spherical sink surfaces SSSS(s) (under the effects of the Earth's rotation and circulations) that are hydraulically developed by any means.

\section{CONFLICTS OF INTEREST}

No conflict of interest was declared by the authors. 


\section{REFERENCES}

[1] Taştan, K., Yıldırım, N., "Effects of intake geometry on the occurrence of a free-surface vortex", Journal of Hydraulic Engineering, 144(4): 04018009, (2018).

[2] Yıldırım, N., Eroğlu, N., Taştan, K., "Occurrences of vortices at an intake of point sink character", Teknik Dergi, 23(1): 5799-5812, (2012).

[3] Taştan, K., Yıldırım, N., "Flow rate along the length of the swirling vortex axis at an intake", Teknik Dergi, https://doi.org/10.18400/tekderg.593595, (2020). (in press)

[4] Denny, D.F., "An experimental study of air-entraining vortices in pump sumps”, Proceedings of the Institution of Mechanical Engineers, 170(2): 106-116, (1956).

[5] Anwar, H.O., Weller, J.A., Amphlett, M.B., "Similarity of free vortex at horizontal intake”, Journal of Hydraulic Research, 16(2): 95-106, (1978).

[6] Jain, A.K., Ranga Raju, K.G., Garde, R.J., "Vortex formation at vertical pipe intake", Journal of the Hydraulics Division, 104(10): 1429-1448, (1978).

[7] Gulliver, S.J., Rindels, A.J., "Weak vortices at vertical intakes”, Journal of Hydraulic Engineering, 113(9):1101-1116, (1987).

[8] Yıldırım, N., Kocabaş, F., "Critical submergence for intakes in open channel flow", Journal of Hydraulic Engineering, 121(12): 900-905, (1995).

[9] Kocabaş, F., Yıldırım, N., "Effect of circulation on critical submergence of an intake pipe", Journal of Hydraulic Research, 40(6): 741-752, (2002).

[10] Yıldırım, N., Akay, H., Taştan, K., "Critical submergence for multiple pipe intakes by the potential flow solution”, Journal of Hydraulic Research, 49(1): 117-121, (2011).

[11] Taştan, K., Yıldırım, N., "Effects of dimensionless parameters on air-entraining vortices", Journal of Hydraulic Research, 48(1): 57- 64, (2010).

[12] Sarkardeh, H., Zarrati, A.R., Roshan, R., "Effect of the intake head wall and trash rack on vortices", Journal of Hydraulic Research, 48(1): 108-112, (2010).

[13] Naderi, V., Farsadizadeh, D., Dalir, A. H., Arvanaghi, H., "Effect of using vertical plates on vertical intake on discharge coefficient", Arabian Journal for Science and Engineering, 39(12): 8627- 8633, (2014).

[14] Suerich-Gulick, F., Gaskin, S., Villeneuve, M., Parkinson, E., "Free surface intake vortices: Theoretical model and measurements", Journal of Hydraulic Research, 52(4): 502-512, (2014).

[15] Taştan, K., "Critical submergence for a horizontal pipe intake", Ain Shams Engineering Journal, https://doi.org/10.1016/j.asej.2020.02.010, (2020). (in press)

[16] Levi, E., "Vortices in hydraulic", Journal of Hydraulic Engineering, 117(4): 399-413, (1991). 